\title{
A photometric pilot study on Sonneberg archival patrol plates
}

\section{How many "constant" stars are in fact long-term variables? ${ }^{\star}$}

\author{
N. Vogt ${ }^{1,2}$, P. Kroll ${ }^{1}$, and E. Splittgerber ${ }^{1}$ \\ 1 Sonneberg Observatory, Sternwartestr. 32, 96515 Sonneberg, Germany \\ e-mail: pk@4pisysteme.de \\ 2 Instituto de Astronomia, Universidad Catolica del Norte, Avenida Angamos 0610, Antofagasta, Chile
}

Receiced 16 March 2004 / Accepted 7 June 2004

\begin{abstract}
The light curves of 216 arbitrarly chosen field stars and of 23 known variables in the Aur/Tau/Ori region were derived ( $7.8 \leq B \leq 12$. 2 ) from scanned, blue-sensitive archival patrol plates, covering a total of 34 years (1961-1995). We achieved a photometric accuracy of $0.07 \ldots 0.12 \mathrm{mag}$ in spite of rather unfavourable locations of most stars near the plate borders. 17 field stars turned out to be variables, most of them with time scales of 1000-8000 days in the form of slow waves with amplitudes between 0.1 and $0.3 \mathrm{mag}$, i.e. below the threshold of traditional variable searches on photographic plates. About $50 \%$ of these new long-term variables exhibit drifts indicating periodic or erratic variability at much longer time scales than covered here. For the 23 known variables we achieved improvements in their periods and amplitudes and detected longterm variations (drifts, waves) in about $50 \%$ of them. The above fraction of low-amplitude long-term variables among field stars implies that a total of about 45000 new variables should be detectable in the Sonneberg patrol plate archive. They will represent a new, hitherto not investigated population of variable stars with a possibly significant impact on our understanding of the stellar interior and evolution.
\end{abstract}

Key words. stars: variables: general - stars: evolution

\section{Introduction}

For more than a century, the photographic plate has been the most reliable light detector and data storage device available for astronomical observations. However, it is being nearly completely replaced by digital detectors like CCDs in practically all astronomical applications, even those requiring wide fields. This implies that most of the older photographic observations are becoming obsolete, unless they document variations on long time scales.

This is the case if one considers the patrol plate archive of the Sonneberg Observatory, which, following Harvard, is the second largest in size and plate number (Bräuer \& Fuhrmann 1992). The Sonneberg Observatory was continuously active in sky patrol observations from the 1930ies until today in a very homogeneous manner, using the same optics and very similar plate scales, sizes and emulsions for many decades. Therefore, the Sonneberg archive which contains more than 275000 plates and films, today represents a unique collection of sky patrol coverage of the entire northern and equatorial sky (down to declination $-33^{\circ}$ ), without any major gaps.

^ Tables 2 and 3 are only available in electronic form at http://www. edpsciences.org
There was a considerable impact of this effort on variable star research. Hoffmeister, together with his collaborators and successors, detected, classified and investigated a total of more than 10000 variable stars in the Milky Way, about 25\% of all variables known at the time when the latest edition of the General Catalogue of Variable Stars (GCVS) was published (Kholopov et al. 1985). The technique used for this purpose: blink comparison and eye estimates of magnitudes. Obviously, only a very small fraction of all the information contained in the plate archive could be extracted this way. Now, with the advent of rapid and precise scanners, the entire plate archive can be digitized and subsequently analysed in a more general manner. As a first step in this direction we determine here the photometric variations of 216 arbitrarily chosen fields stars and, in addition, 23 known variables over a period of 34 years. Our study is restricted to rather small areas (about 74 square degrees in total) in the region of Aur/Tau/Ori. The main aim of this patrol project is to determine the photometric accuracy of scanned stellar images on patrol plates, as well as to find out what kind of information on the long-term variability of all classes of stars can be derived and expected from a comprehensive analysis of patrol plates. 


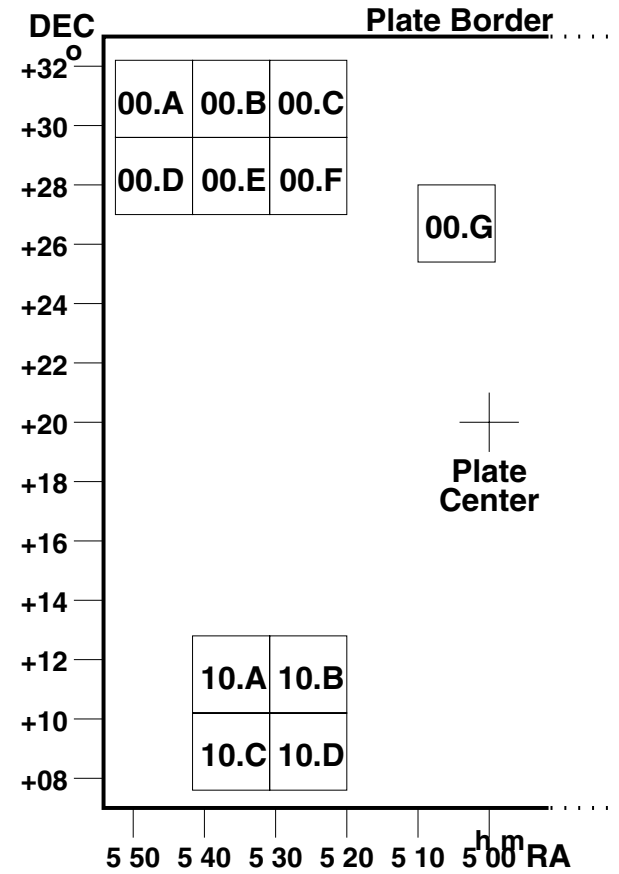

Fig. 1. Approximate locations of the sub-fields on the SSP field $5 \mathrm{~h}+20^{\circ}$ (plate center).

\section{Plate material, scanning and reduction procedure}

The Sonneberg Sky Patrol (SSP), originally proposed by Paul Guthnik (1879-1947), is recording the entire accessible sky with 14 short-focus cameras simultaneously in two colours "pg" and "pv" (Bräuer \& Fuhrmann 1992; Bräuer et al. 1999). The plate size is $13 \times 13 \mathrm{~cm}^{2}$, the scale $830^{\prime \prime} / \mathrm{mm}$ resulting in a useful field size of about $26^{\circ} \times 26^{\circ}$. The cameras are centred at declinations $-20^{\circ}, 0^{\circ},+20^{\circ},+40^{\circ},+60^{\circ}$ and $+80^{\circ}$, recording the sky every $1^{\mathrm{h}}$ in right ascension at $\delta=+40^{\circ}$ and south of it, and every $2^{\mathrm{h}}$ in the $60^{\circ}$ and $80^{\circ}$ zones. The limiting magnitudes are of the order of $14.5 \mathrm{~m}$ ing (blue) and 13.5 in pv (red), but the average limit achieved is about $1 \mathrm{mag}$ brighter than these values.

Plate scanning was performed with the digitalization machine DIA ("Digital Image Analyser") described by Kroll \& Neugebauer (1993). DIA was able to scan an SSP plate with a resolution of $15 \mu \mathrm{m}$ and 8 bit data depth within $45 \mathrm{~min}$. More than 5000 plates have been recorded this way between 1994 and 1999, mainly in the Taurus/Orion region for the study of $\mathrm{T}$ Tauri stars (Heines 1999). Each plate was subdivided into 4 quarter subsections for the scanning procedure, called 00 , 01, 10 and 11 for the NE, NW, SE and SW quarter resp. of each plate.

For our pilot project we selected a total of 11 smaller subfields (about $2.8^{\circ} \times 2.8^{\circ}$ ) in the quarter sections 00 and 10 of the SSP field $5^{\mathrm{h}}+20^{\circ}$. The 7 fields in quarter 00 are called: 00.A, 00.B, 00.C , .. 00.G, the remaining 4 in quarter 10: 10.A, 10.B, 10.C and 10.D. These sub-fields were chosen in order to include some known bright variable stars. Their positions on the sky and plate are shown in Fig. 1 in a schematic way.
Most of them are rather far away from the plate centre and near the edges and/or corners of the plate. This choice was made to study the photometric accuracy under "worst case conditions". Our study refers only to the blue pg plates.

In each of these sub-fields 20-30 field stars were selected according to the following criteria:

1. accurate $B$ and $B-V$ values are available from the HIPPARCOS and TYCHO photometric catalogue (van Leeuwen et al. 1997);

2. Single star image on the SSP plates, well separated from its neighbour, no blends;

3. There is no strong background variation present in the surroundings of the scanned star image;

4. The entire magnitude range of $7.8 \leq B \leq 12$. 2 and colours from $B-V=0.0-1.8$ or more is represented in the sample.

For the measuring procedure we used the software package described by Kroll \& Neugebauer (1993). The basic algorithm of this method is to perform a seven-parametric, three-dimensional Gaussian fit over the pixel grey values of a single star imprint. It was shown that the logarithm of the volume, $I=\ln V$, of the Gaussian bell is a convenient measure of the magnitude of the star. This software, however, requires the manual selection and measurement of each star image on each of the nearly 500 SSP plates, a task which was performed by ES and NV, eliminating, this way, also contamination by emulsion faults, scratches, spots, as well as meteor, satellite and airplane tracks and other artefacts (see, e.g. Kroll 1999).

For each sub-field, the $I$-values were linked to the HIPPARCOS and TYCHO catalogue magnitudes, $m_{B}$ and $m_{V}$, of the constant stars in order to define a transformation between $I$ and the photographic magnitude $m_{\text {phot }}$ :

$m_{\mathrm{phot}}=a_{0}+a_{1} I+a_{2} I^{2}+a_{3}\left(m_{B}-m_{V}\right)$.

By setting $m_{\text {phot }}=m_{B}$, for each sub-field and each plate, the coefficients, $a_{0} \ldots a_{3}$, were determined with the least-square method. This fit also gives the standard deviation $\sigma$ corresponding to the differences between catalogue magnitudes and reduced magnitudes of all stars in a sub-field. In order to apply the above fit to unknown program stars one has to know their $B-V$ colour. In a few cases in which this colour value was not known we applied a mean value of $B-V=0.6$. If the colour is different from this, some zero point shift in $m_{\text {phot }}$ will result. This, however, will not affect the variability discussion given here.

Originally, we had included stars between $6^{\mathrm{m}}$ and $13^{\mathrm{m}}$ in our measurement program. In the course of the reduction procedure the coverage of very bright and very faint stars in most sub-fields was too poor to get reliable photometric values. In addition, systematic variations from the above parabolic fit arise as soon as the total magnitude range exceeds about $4.5 \mathrm{mag}$. Therefore, we limited the final reduction to stars in the range $7.8 \leq m_{B} \leq 12$. 2 .

The colour coefficients $a_{3}$ were determined, in a first step, for different sets of about 60 plates each taken within a three year interval. However, the coefficient did not vary significantly from epoch to epoch, so we could use a mean colour 
Table 1. Center distances, colour coefficients and mean scatter $\sigma$ of the sub-fields.

\begin{tabular}{cccc}
\hline \hline Sub-field & $\begin{array}{c}\text { Distance from } \\
\text { plate center } \\
\text { (degrees) }\end{array}$ & $\begin{array}{c}\text { Colour } \\
\text { coefficient }\end{array}$ & $\begin{array}{c}\text { Mean standard deviation } \\
\text { from calibration fit } \\
\sigma \text { (mag) }\end{array}$ \\
\hline 00.G & 6.5 & 0.359 & 0.077 \\
10.B & 10.4 & 0.356 & 0.087 \\
00.F & 10.6 & 0.353 & 0.091 \\
00.E & 12.4 & 0.249 & 0.098 \\
10.A & 12.7 & 0.281 & 0.083 \\
00.C & 12.8 & 0.309 & 0.120 \\
10.D & 12.8 & 0.336 & 0.112 \\
10.C & 14.1 & 0.277 & 0.088 \\
00.B & 14.3 & 0.305 & 0.110 \\
00.D & 14.4 & 0.289 & 0.089 \\
00.A & 16.2 & 0.422 & 0.096 \\
\hline
\end{tabular}

coefficient for each sub-field. Table 1 lists their values, together with the approximate mean distance of each sub-field from the plate center. Apparently, there is no correlation; however, the most distant field 00.A has the largest value of $a_{3}$.

The mean distances from the plate center and the colour coefficients for each sub-field are listed in Table 1, together with the mean standard deviations $\sigma$ from the calibration fits.

They range from 0.07 to $0.12 \mathrm{mag}$, and there is no close correlation with the position on the plate. However, the field nearest to the plate center (00.G) also reveals the smallest scatter, as expected. In general, we derived our photometric data with a mean error of $0.096 \mathrm{mag}$, and we expect that this value could improve to about $0.06-0.08$ mag if all stars of an entire plate are measured, since most stars in our sample are located near the plate edges.

\section{Constant stars and new variables}

The above reduction procedure reveals light curves of a total of 239 stars, with an average of about 450 measurements distributed more or less homogeneously over 34 years (1961-1995). 23 of them are known variables that will be discussed in Sect. 4.

All light curves were analysed in various ways. We calculated seasonal means and searched for drifts, waves and erratic variability in all accessible time scales. In addition, we applied a period search routine developed by Schwarzenberg-Czerny (1989), searching for periodicities between 1 and 10000 days. A critical comparative analysis of all light curves revealed a total of 17 new variable stars while the remaining 199 stars of our sample have to be considered as constant within the time interval and accuracy considered here. The latter are listed in Tables 2 and 3 (see online material). The scatter around their mean magnitude value is always of the order of 0.08-0.12 mag, as expected from the photometric accuracy determined in Sect. 2. Only these constant stars were used for the magnitude calibration.

The most important properties of the 17 new variable stars are given in Table 4. Eight of them were drifting, showing a linear increase or decrease in brightness over the entire 34-year time interval with a total amplitude between 0.09 and 0.25 mag (- sign refers to increasing, + sign to decreasing brightness with time). This may indicate the presence of periodic or erratic variations at much larger time scales than covered here. Five stars show periodic long-term variations with periods between 3500 and 11000 days, and amplitudes up to 0.25 mag. In addition, in seven cases erratic variability with similar or shorter time scales (down to about 20 days) is present while in one case (S10953 = GSC 708.0904) the enhanced scatter suggests unresolved short-term variability. The amplitude of the erratic variations ranges from 0.15 to $0.5 \mathrm{mag}$. A special case is $\mathrm{S} 10955=$ GSC 714.0246 for which the period search routine has revealed a strictly periodic variation with 1.58625 days, displaying the typical light curve of an eclipsing binary (Fig. 4), probably of $\beta$ Lyrae type with small amplitude ( $0.2 \mathrm{mag}$ ).

The most interesting light curves of these new variables are shown in Figs. 2 to 9, together with that of a nearby constant star from the same sub-field and similar brightness.

In general, most observed amplitudes of the new variables do not exceed $0.3 \mathrm{mag}$, the typical threshold for a detection with blink comparator or similar visual inspection methods, as applied traditionally at the Sonneberg Observatory. Therefore, it is not surprising that the variables listed in Table 4 have previously not been detected. However, four of them are listed in the New Catalogue of Suspected Variables (NSV: Kukarkin et al. 1982).

The spectral type o the 17 new variable stars are distributed as follows: $\mathrm{B}(4$ stars $), \mathrm{A}(8), \mathrm{F}(1), \mathrm{G}(0), \mathrm{K}(1)$ and $\mathrm{M}(3)$. This could be a hint of a bimodal frequency distribution in spectral types of long-term variables with low amplitude, with maxima around types $\mathrm{A}$ and $\mathrm{M}$, since the fraction of A type among the constant stars in our sample is only $20 \%$, that of M stars only $1 \%$. The maximum at A stars is surprising and would require more data for confirmation. This preliminary result suffers from the low star numbers.

\section{Known variable stars}

The 23 previously named variable stars included in our study are listed in Table 5; a sample of the most interesting light curves is given in Figs. 10 to 20.

These variables can be subdivided into the following classes (GCVS designations in brackets):

1. Eclipsing binaries of Algol type (EA): 5 stars;

2. Mira-type variables (M): 2 stars;

3. Other red semiregular or irregular giant/supergiant late type variables (LB,LC,SR,SRA): 6 stars;

4. Orion-type variables (INS,INA): 5 stars;

5. Be and shell stars (BE,GCAS): 4 stars;

6. $\delta$ Scuti variable (DSCT): 1 star.

In 4 of the 5 eclipsing binaries our data confirm or are compatible with the published ephemeris. Only for FP Aur is this 
Table 4. New variable stars.

\begin{tabular}{|c|c|c|c|c|c|c|c|c|c|c|}
\hline $\begin{array}{l}\text { Sonneberg } \\
\text { variable } \\
\text { designation }\end{array}$ & $\begin{array}{l}\text { Hip or } \\
\text { GSC No. }\end{array}$ & $\begin{array}{l}\text { Spec. } \\
\text { type }\end{array}$ & $N$ & $\begin{array}{r}\text { Mean } \\
\text { mag } \\
B\end{array}$ & $\begin{array}{l}\text { Drift } \\
\text { ampl. } \\
\text { (mag) }\end{array}$ & $\begin{array}{l}\text { Periodic } \\
P(\mathrm{~d})\end{array}$ & $\begin{array}{l}\text { Var. } \\
\text { ampl. } \\
\text { (mag) }\end{array}$ & $\begin{array}{l}\text { Erratic Varia } \\
\text { Time scale } \\
\text { (d) }\end{array}$ & $\begin{array}{l}\text { ble } \\
\text { ampl. } \\
\text { (mag) }\end{array}$ & Remarks \\
\hline S10949 & Hip 25972 & A0 & 478 & 8.547 & -0.15 & & & $30-8000$ & 0.35 & \\
\hline S10950 & Hip 26162 & B8e & 467 & 8.570 & -0.22 & & & $1000-4000$ & 0.5 & \\
\hline S10951 & Hip 26669 & A0 & 497 & 9.027 & & 8000: & 0.2 & $30-300$ & 0.15 & see Fig. 2 \\
\hline S10952 & Hip 27699 & B5e & 477 & 9.010 & +0.26 & 4700 & 0.25 & & & see Fig. 3 \\
\hline S10953 & 0708.0904 & M0 & 473 & 11.158 & & & & & & $=\mathrm{NSV} 2001,(1), \sigma=0.186 \mathrm{mag}$ \\
\hline S10954 & 0709.0746 & A5 & 425 & 9.778 & +0.21 & & & & & \\
\hline S10955 & 0714.0246 & A0 & 481 & 9.281 & & 1.58625 & 0.2 & & & (2), see Fig. 4 \\
\hline S10956 & 0714.1144 & K0 & 476 & 9.578 & & 11000: & 0.2 & & & "wave" see Fig. 5 \\
\hline S10957 & 1873.0775 & M & 468 & 11.026 & & & & $4000-6000$ & 0.3 & $=$ NSV 2444, Fig. 6 \\
\hline S10958 & 1875.2587 & A2 & 474 & 8.182 & +0.18 & & & & & $=\mathrm{NSV} 2670$ \\
\hline S10959 & 2403.0702 & $\mathrm{~A} 5 \mathrm{~m}$ & 461 & 10.265 & +0.17 & 8000: & 0.15 & & & see Fig. 7 \\
\hline S10960 & 2405.0168 & A0 & 461 & 10.764 & +0.09 & & & & & \\
\hline S10961 & 2405.0203 & $\mathrm{~F} 2$ & 389 & 11.370 & & & & 2000 & 0.25 & \\
\hline S10962 & 2405.1545 & B0e & 409 & 9.716 & & 3580 & 0.12 & & & see Fig. 8 \\
\hline S10963 & 2407.0022 & M3 & 372 & 11.554 & & & & $20-100$ & 0.4 & $=$ NSV 2073 \\
\hline S10964 & 2408.0661 & B5 & 397 & 9.003 & & & & 3000 & 0.2 & see Fig. 9 \\
\hline S10965 & 2409.0265 & A2 & 401 & 11.091 & -0.16 & & & & & \\
\hline
\end{tabular}

(1) Short-term variable, only enhanced scatter, no significant long-term variability.

(2) New eclipsing binary, probably of EB type ( $\beta$ Lyrae); epoch of minimum: HJD 24 37585.82. Possible alias period: 1.59315.

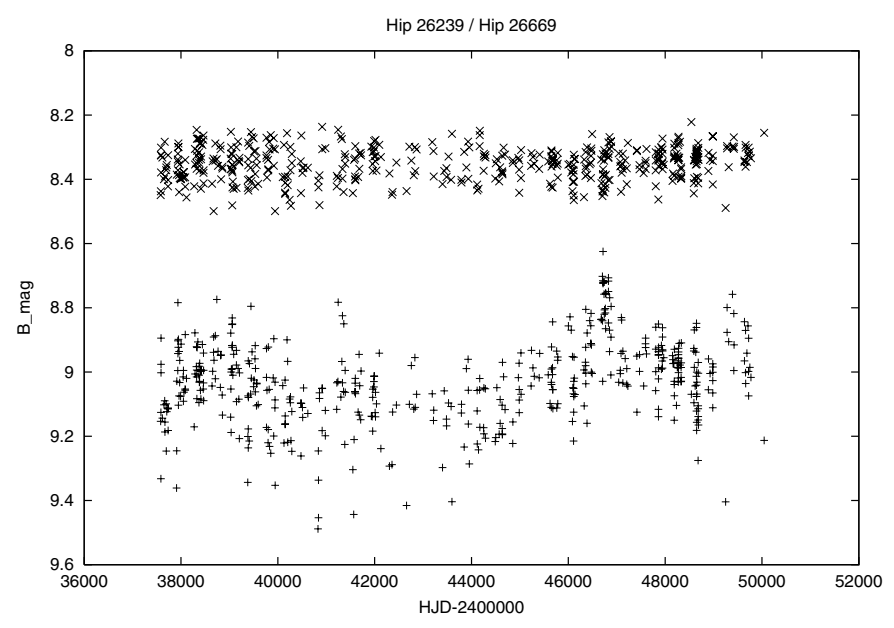

Fig. 2. Light curve of the new variable star S10951 = Hip 26669 (lower panel) and the constant star Hip 26239.

not the case: The GCVS gives a period of 0.947236 and an amplitude of only $0.3 \mathrm{mag}$. Our data give a mean brightness of $\bar{B}=11$ m 419 in HJD 2437500 to $41700(\sigma=0.15 \mathrm{mag})$ and $\bar{B}=11$. 640 in HJD 2442400 to 49800 ( $\sigma=0.28 \mathrm{mag})$, but no indication of eclipses. The enhanced scatter may be due to another kind of short-term variability.

For one Mira star, U Aur, our data give exactly the published ephemeris values. In the other case, BK Ori, the GCVS gives a period of 354.2, valid after HJD 2438800, and an epoch of maximum HJD 2440925. At earlier epochs, the

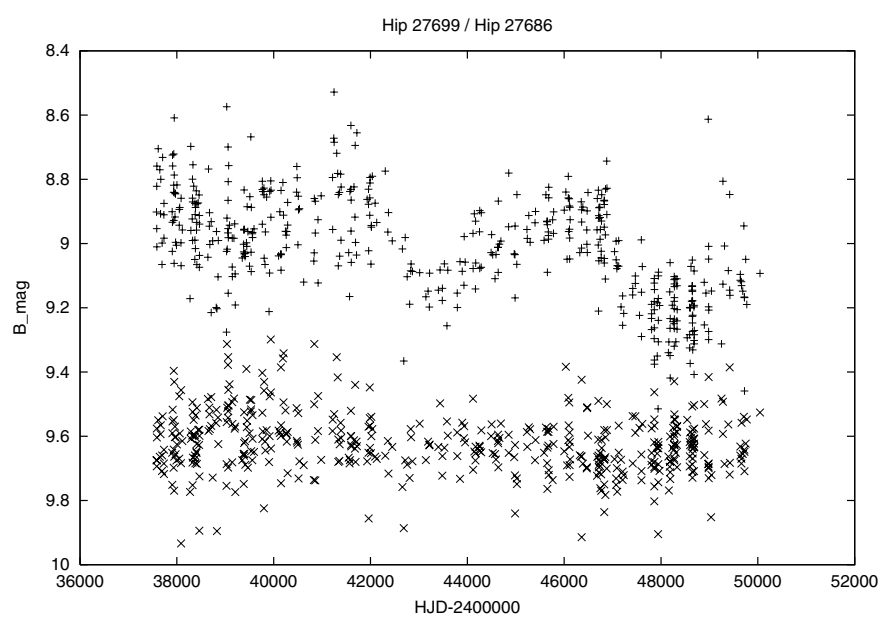

Fig. 3. Light curve of the new variable S10952 = Hip 27699 (upper panel) and the constant star Hip 27686.

period varied between 326 and 346 days. Our data fit best the element maximum = HJD $2440908+346.3 \mathrm{E}$ (see Fig. 13).

As expected, the third group of late type giants and supergiants demonstrate a wide range of behaviour, from unresolved short-term variability (V440 Ori), to erratic variations at all time scales from 20 to 3000 days and amplitudes up to 0.4 mag, as well as possible long-term waves with quasi-periods up to 8000 days. A special case is AB Tau: the GCVS gives an epoch HJD 2437340 for the light maximum and a period of 142.0 . Our data fit well to the epoch, but the period search 
Table 5. Known variable stars.

\begin{tabular}{|c|c|c|c|c|c|c|c|c|c|c|}
\hline \multirow[t]{2}{*}{ Name } & \multirow{2}{*}{$\begin{array}{l}\text { Type } \\
\text { (GCVS) }\end{array}$} & \multirow{2}{*}{$\begin{array}{l}\text { Spectr. } \\
\text { type }\end{array}$} & \multirow[t]{2}{*}{$N$} & \multirow{2}{*}{$\begin{array}{r}\text { Mean } \\
\text { mag. } \\
B\end{array}$} & \multirow{2}{*}{$\begin{array}{l}\text { Drift } \\
\text { ampl. } \\
\text { (mag) }\end{array}$} & \multicolumn{2}{|c|}{ Periodic Variable } & \multicolumn{2}{|c|}{ Erratic Variable } & \multirow[t]{2}{*}{ Remarks } \\
\hline & & & & & & $P(\mathrm{~d})$ & $\begin{array}{l}\text { ampl. } \\
\text { (mag) }\end{array}$ & $\begin{array}{l}\text { Time scale } \\
\text { (d) }\end{array}$ & $\begin{array}{l}\text { ampl. } \\
\text { (mag) }\end{array}$ & \\
\hline U Aur & $\mathrm{M}$ & M9 & 392 & 11.184 & & 408.09 & 2.5 & & & (4), see Fig. 10 \\
\hline RZ Aur & $\mathrm{EA} / \mathrm{SD}$ & $\mathrm{A}$ & 306 & 11.564 & & 3.010644 & $?$ & & & (1), (3) \\
\hline FP Aur & EA & & 286 & 11.531 & +0.35 & $0.947236(?)$ & & & & (5), see text \\
\hline FU Aur & LB & CII & 426 & 11.152 & & & & $20-100$ & 0.4 & carbon star \\
\hline FW Aur & EA/SD & & 322 & 11.671 & & 2.55997 & $?$ & & & $(1),(2),(3)$ \\
\hline HH Aur & INSB: & G6IV & 485 & 9.95 & & & & & & constant, $\sigma=0.104^{m}$ \\
\hline V356 Aur & DSCT & F4IIIp & 483 & 8.69 & & 0.18916 & & & & constant, $\sigma=0.139^{m}$ \\
\hline V362 Aur & $\mathrm{LC}$ & M1.5Ia & 438 & 9.612 & +0.23 & & & $1000-3000$ & 0.4 & see Fig. 11 \\
\hline V399 Aur & SR & $S$ & 431 & 11.734 & & 8000: & 0.5 & $30-1000$ & 0.3 & (8) \\
\hline V438 Aur & GCAS & B2pshl & 415 & 8.044 & & & & $50-6000$ & 0.5 & see text and Fig. 12 \\
\hline BK Ori & M & M7 & 165 & 11.899 & & 346.3 & $>2.5$ & & & (1), (6), see text and Fig. 13 \\
\hline CO Ori & INSB & G5Vpe & 404 & 11.711 & -0.50 & & & $20-1000$ & 0.8 & (1) \\
\hline GW Ori & INST & $\mathrm{K} 3 \mathrm{~V}: \mathrm{e}$ & 476 & 10.818 & +0.14 & & & & & \\
\hline HK Ori & INSA & A4pe & 426 & 11.608 & +0.13 & 2400 & 0.25 & $30-1000$ & 0.2 & (8), see Figs. 14 and 15 \\
\hline OS Ori & $\mathrm{EA} / \mathrm{SD}$ & A0 & 358 & 12.087 & & 2.383525 & $>0.6$ & & & (1), (3), (10), see Fig. 16 \\
\hline V440 Ori & $\mathrm{LB}$ & M5 & 357 & 11.692 & +0.13 & & & & & $(10), \sigma=0.32^{m}$ \\
\hline V451 Ori & GCAS & B9 & 425 & 9.858 & -0.09 & & & $100-3000$ & 0.1 & \\
\hline V1374 Ori & $\mathrm{BE}$ & B8 & 481 & 8.082 & -0.25 & 5685 & 0.5 & & & (8), (9), see Fig. 17 \\
\hline V1376 Ori & LB & M5 & 483 & 9.029 & & see (11) & see (11) & & & $=\mathrm{NSV} 2258,(11)$ \\
\hline V1409 Ori & INA & AIab:e & 471 & 10.490 & & 3445: & $0.1:$ & & & $=$ =NSV 2041, (8) \\
\hline SV Tau & $\mathrm{EA} / \mathrm{SD}$ & B9 & 467 & 10.128 & & 2.1669051 & 1.2 & & & (4), (7) see Fig. 18 \\
\hline AB Tau & SRA & M3 & 456 & 11.649 & & 143 & 0.25 & $10-50$ & 0.2 & (6), see text and Fig. 19 \\
\hline V1163 Tau & $\mathrm{BE}$ & B1Vne & 469 & 8.451 & & & & $20-6000$ & 0.5 & see Fig. 20 \\
\hline
\end{tabular}

(1) Minimum magnitude below threshold of Sonneberg patrol plate.

(2) Observations in the phase interval $0.95-1.03$ are missing.

(3) GCVS period compatible with our data.

(4) GCVS period confirmed by our data.

(5) GCVS period not compatible with our data.

(6) GCVS period modified and/or improved by our data.

(7) Low-amplitude secondary minimum near phase 0.5 .

(8) New period, derived from our data.

(9) Epoch of maximum HJD 2436820.

(10) Enhanced scatter, unresolved short-term variability.

(11) Possible periods $436^{\mathrm{d}}, 61^{\mathrm{d}} .82$ and $41^{\mathrm{d}} 37$; amplitudes $0.1-0.15 \mathrm{mag}$.

routine reveals $143^{\mathrm{d}}$ as the best period. The enhanced scatter (see Fig. 19) implies additional short-term variability.

One of the Orion-type variables (HH Aur) turned out to be constant in our data set. The remaining variables show either erratic variations at time scales of 20 to 1000 days with amplitudes up to $0.8 \mathrm{mag}$, or waves with quasi-periods of 2400-3500 days and amplitudes up to $0.25 \mathrm{mag}$.

The group of Be stars is characterized by erratic variations in all time scales between 20 and 6000 days, with amplitudes up to $0.6 \mathrm{mag}$. The only $\delta$ Scuti star in our sample, V 356 Aur, seems to be constant with a slightly enhanced scatter. Its 0.19 -period (amplitude $0.1 \mathrm{mag}$ ) reported in the literature apparently was not resolved by our data.
Due to our period search routine it was possible to discover new, hitherto unknown long-term periodicities in four cases (V399 Aur, HK Ori, V 1374 Ori and V 1409 Ori), with $2400^{\mathrm{d}} \leq$ $P \leq 8000^{\mathrm{d}}$ and amplitudes between 0.1 and $0.5 \mathrm{mag}$. In another four cases (U Aur, BK Ori, SV Tau and AB Tau) we were able to modify and/or improve the published periods. In addition, eight stars (35\% of our sample of previously known variables) show significant drift variations in the entire 34 year interval covered, with amplitudes between 0.09 and $0.5 \mathrm{mag}$. Three of them belong to the group of Orion variables, the remaining ones are more or less evenly distributed among the other variable types. All these findings support the importance of this kind of supplementary information which can be 


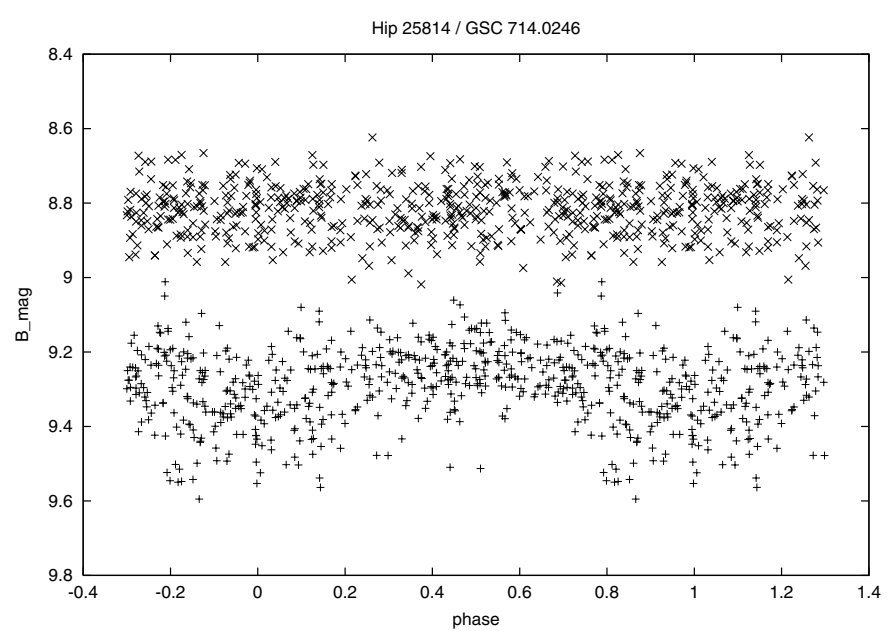

Fig. 4. Light curve of the new eclipsing binary S10955= GSC 714.0246 (lower panel) and the constant star Hip 25814 vs. phase of the period 1.58625 .

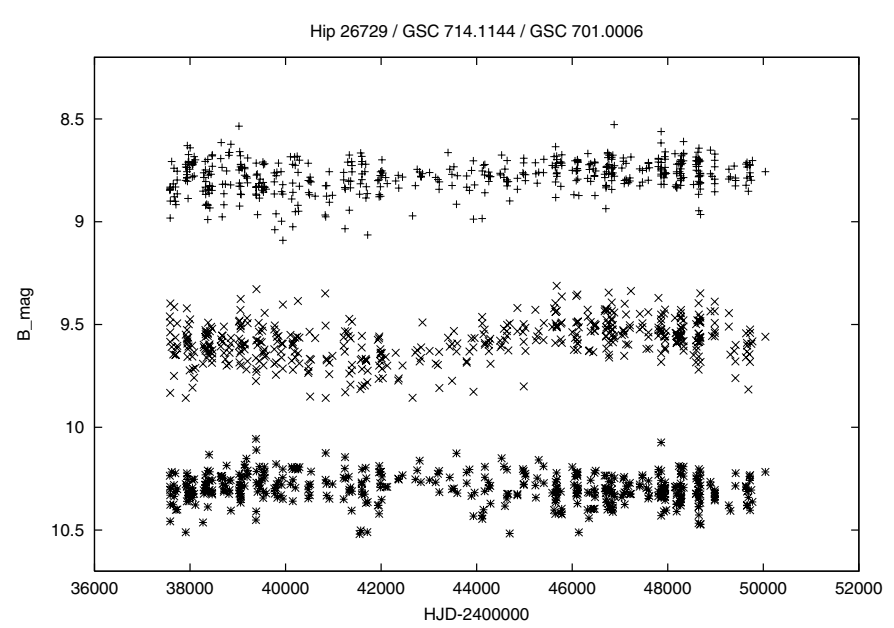

Fig. 5. Light curve of the new variable S10956 = GSC 714.1144 (central panel) and the two constant stars Hip 26729 (upper panel) and GSC 701.0006 (lower panel).

derived by a study like ours even for known variables: they all are either well known, or recently discovered by HIPPARCOS due to striking short-term variations. Their long-term behaviour has never been investigated because there is essentially no way to do this other than via sky patrol plate archives.

\section{Discussion}

Our sample consisted of a total of 316 field stars and 23 known variables. 17 of the field stars, i.e. $7 \%$, are variable with amplitudes between 0.1 and $0.3 \mathrm{mag}$, below the threshold of traditional visual searches on photographic sky patrol plates. Richter (1968) estimated that $2 \%$ of the bright field stars of about $6^{\mathrm{m}}$ show variability exceeding the above threshold in amplitude while this fraction is a factor of 10 lower $(0.2 \%)$ for $16^{\mathrm{m}}$ stars. He explained this difference by the fact that bright stars are mainly giants which have a much stronger tendency to vary while in the faint star sample relatively stable main sequence stars are dominating. On the other hand,

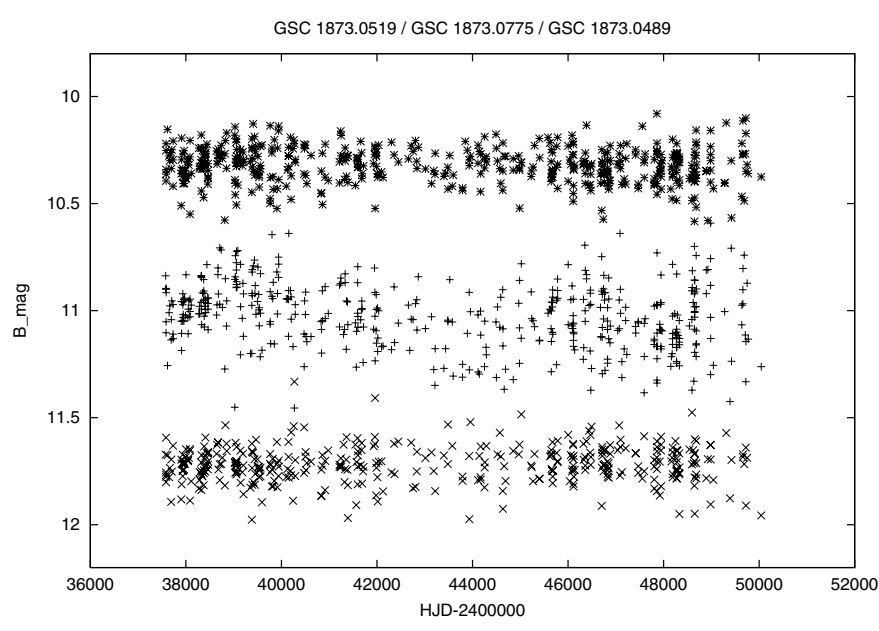

Fig. 6. Light curve of the new variable S10957 = GSC 1873.0775 (NSV 2444: central panel) and the two constant stars GSC 1873.0519 (upper panel) and GSC 1873.0489 (lower panel).

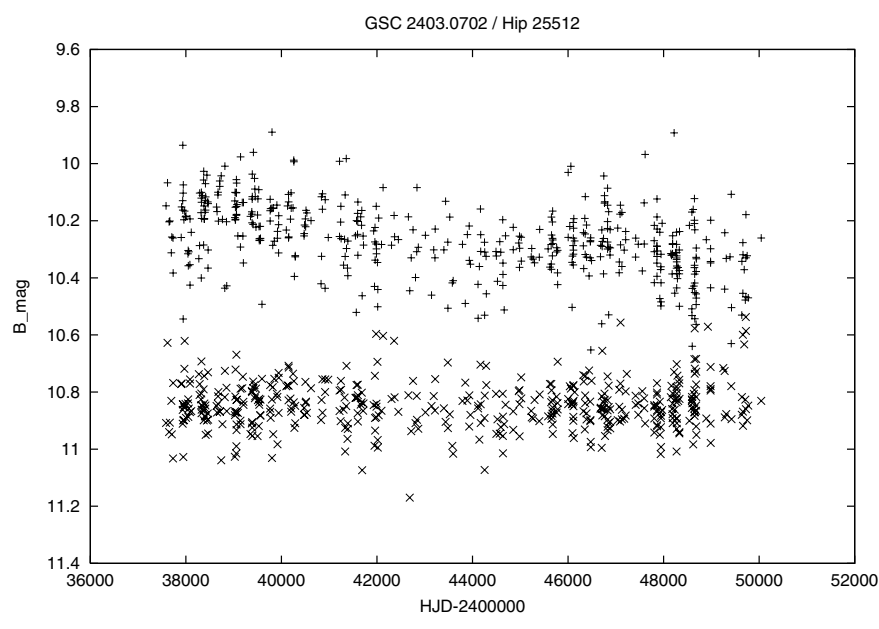

Fig. 7. Light curve of the new variable S10959 = GSC 2403.0702 (upper panel) and the constant star Hip 25512.

Jackisch (1963) found in a photoelectric study on microvariability that about $40 \%$ of supergiants, $26 \%$ of giants and $16 \%$ of main sequence stars show variations with amplitudes of more than 0.02 mag. Our fraction of $7 \%$ lies between these earlier findings, as is the amplitude range covered by us, which links those of Richter (1968) and Jackisch (1963).

This comparison, however, has to be considered with caution for two reasons. Firstly, our fraction of $7 \%$ variables is a lower limit because many "constant" stars show drifts and/or possible waves with amplitudes between 0.05 and $0.1 \mathrm{mag}$ which, however, failed the test of statistical significance with the presently available data. This problem will be solved as soon as the red plates are included in the analysis. They will provide a simultaneously observed, independent data set in a band pass whose variations should be similar to those on blue plates. Secondly, none of the above cited studies investigated the long-term behaviour. 15 of the 17 new variables show variations with time scales of the order of 1000 days or longer. This makes any comparison with published results difficult.

Similar arguments are valid if we compare our study with those made with other modern techniques. Recently, many 


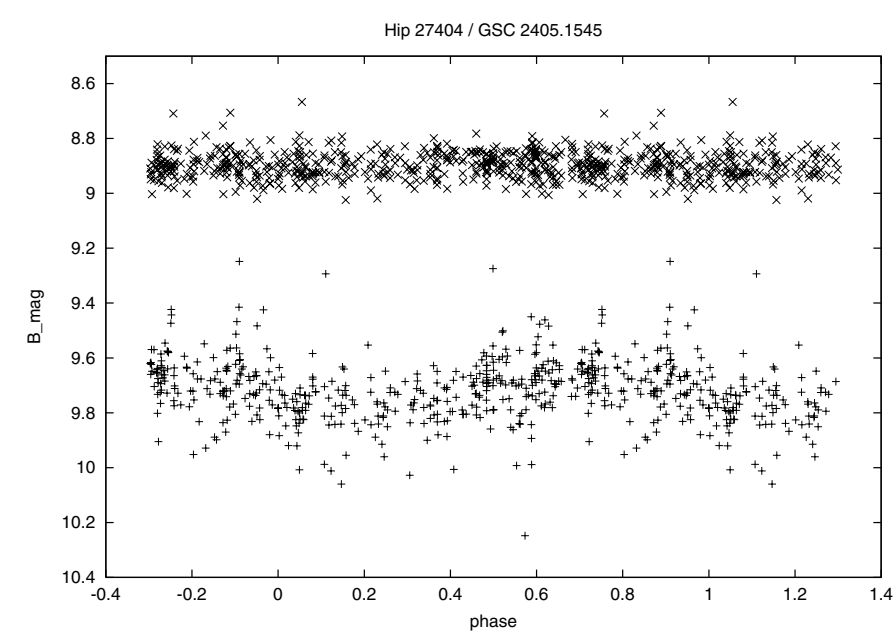

Fig. 8. Light curve of the new periodic variable $\mathrm{S} 10962=$ GSC 2405.1545 (lower panel) and the constant star Hip 27404 vs. phase of the period 3580 days.

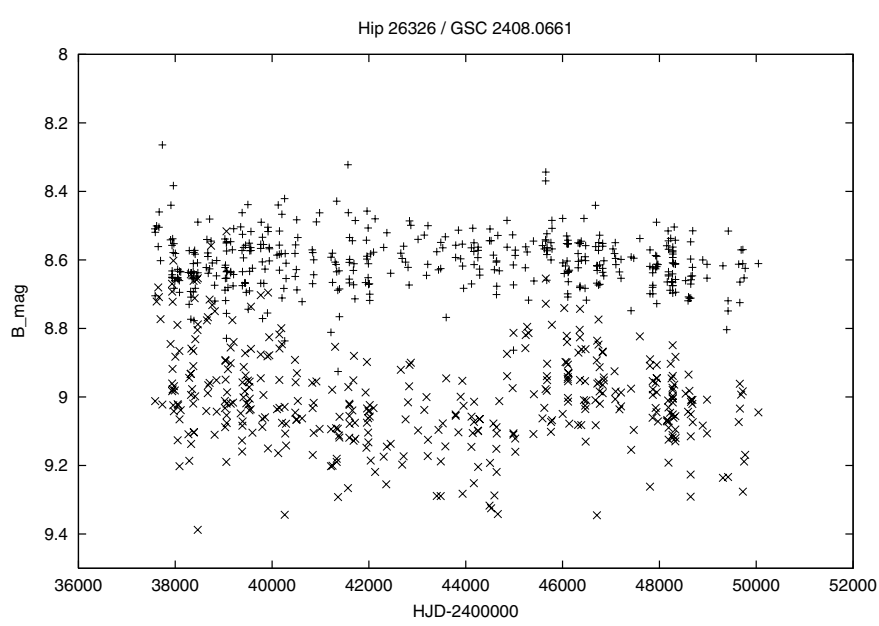

Fig. 9. Light curve of the new variable S10964 = GSC 2408.0661 (lower panel) and the constant star Hip 26326.

new variable stars have been detected and investigated as a by-product of the search for gravitational microlensing effects such as MACHO and OGLE. These surveys record simultaneously millions of stars on large CCD arrays with higher photometric accuracy, lower limiting magnitude and better time resolution than provided by SSP. OGLE has detected a total of about 200000 variables stars in the Galactic Bulge (Wozniak et al. 2002) and 68000 variables in the Magellanic Clouds (Zebrun et al. 2001). Thus, the question arises to what extent we should study relatively bright stars on photographic sky patrol plates (with all the problems of calibration, image distortions, blends etc.) if we can get similar information from other ongoing research projects?

We would have to wait more than 50-100 years to be able to cover the time scales available in Harvard, Sonneberg and few similar existing plate archives. Archival studies cover mainly stars of the solar neighbourhood, i.e. a rather homogeneous population of stars whose fundamental data such as spectral type, luminosity class, radial velocity, parallax, proper

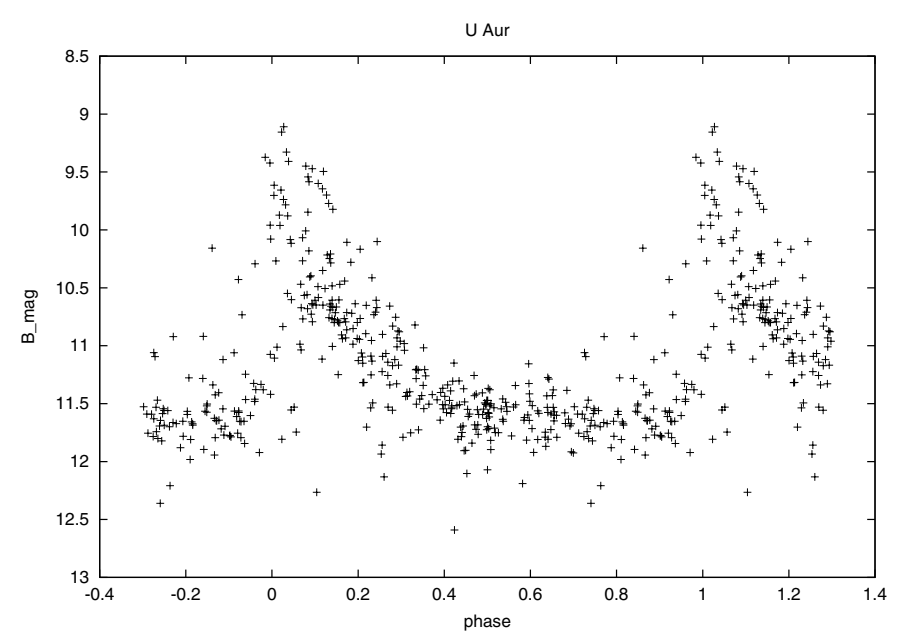

Fig. 10. Light curve of the Mira type variable U Aur vs. phase of the period 408.09 .

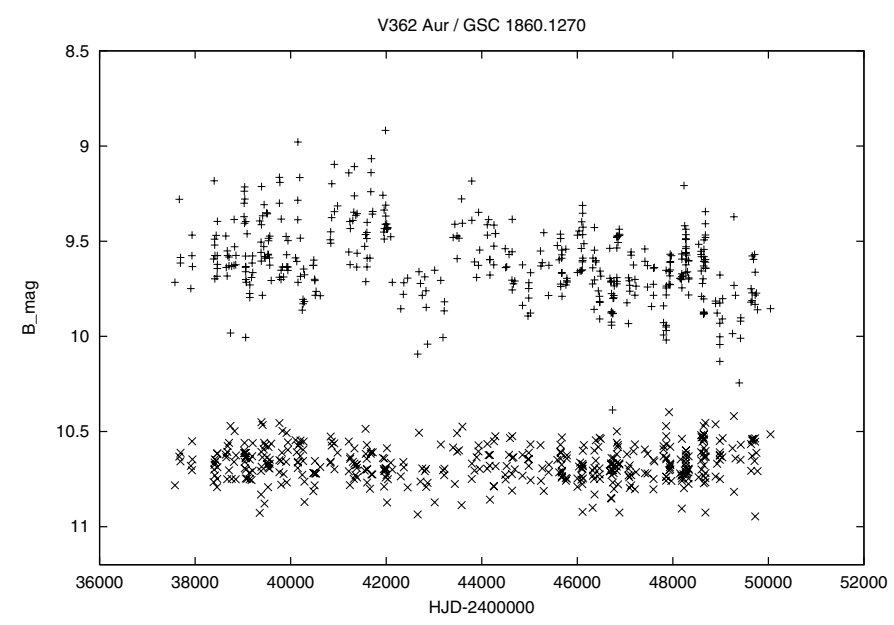

Fig. 11. Light curve of the LC type variable V362 Aur (upper panel) and the constant star GSC 1860.1270.

motion, UV and IR spectrum etc. are known or at least will be known very soon due to scheduled survey projects and space missions. In contrast, OGLE and MACHO observe a mixture of stellar populations at far distances, consisting of very faint stars without any hope of easily obtaining the fundamental data mentioned above and required for a meaningful astrophysical discussion of their variation. Both methods, instead, could complement each other. In the near future plates will definitely be replaced by large CCDs, but the policy to observe the entire sky or at least a large fraction of it should be maintained.

The future impact of a complete analysis of all SSP plates is obvious: according to Allen (1973) there are about 29 stars per square degree brighter than $12^{\mathrm{m}} 0$ in average. SSP has covered the entire sky down to about $-30^{\circ}$ declination, containing about 28000 square degrees in total. This means that a total of roughly 800000 stars in the magnitude range of our pilot study have been monitored in Sonneberg during the past five or more decades. These data will soon be available in digital form: about $30 \%$ of the plates have already been scanned with the new HP flat-bed scanners, and we expect to digitize 


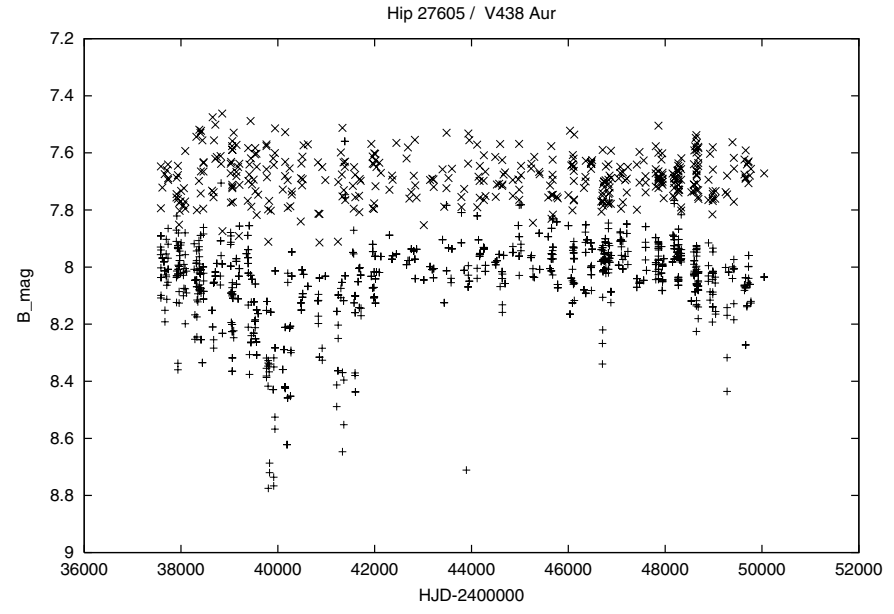

Fig. 12. Light curve of the $\gamma$ Cas type variable V438 Aur (lower panel) and the constant star Hip 27605.

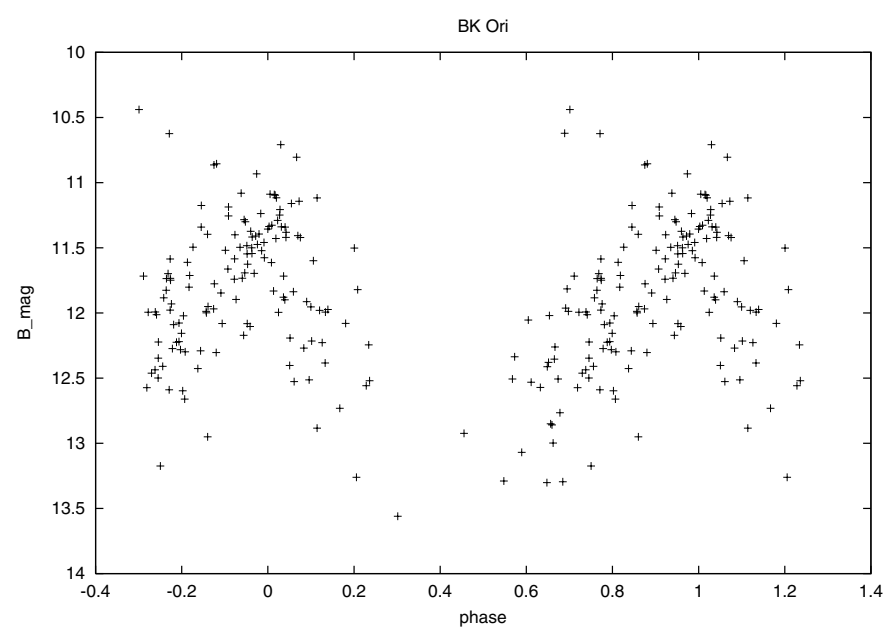

Fig. 13. Light curve of the Mira type variable BK Ori vs. phase of the period 346.3 .

the entire Sonneberg archive within the next five years. If we modify our software so that full-automatic photometric measurements are possible and if we eliminate, in a first attempt, $20 \%$ of the targets due to blends, background variability etc. we could study the long-term behaviour of about 650000 stars just with the methods presented here. $7 \%$ of them, i.e. about 45000 are expected to show long-term variability according to our pilot results; this number is of the same order as that of all known galactic variable stars in the GCVS. This means, on the other hand, that more than $50 \%$ of stellar variability is unknown to us because never investigated with proper methods. We will be able to determine, for the first time, the entire frequency distribution of variable stars, including its hitherto unknown tail at low amplitudes and time scales of over 1000 days. Such a study will have important consequences for our understanding of the stellar interior, evolution and variability.

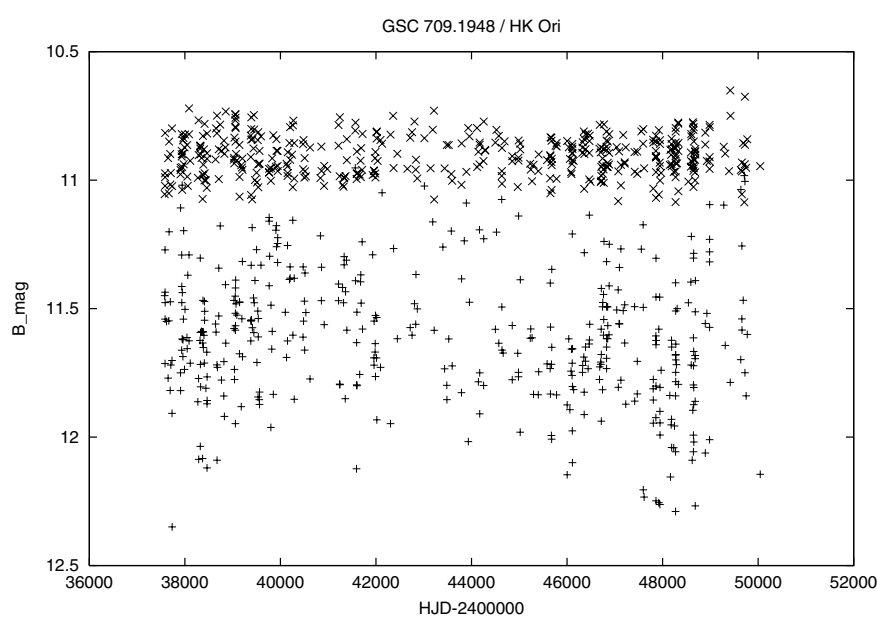

Fig. 14. Light curve of the INSA type variable HK Ori (lower panel) and the constant star GSC 709.1948.

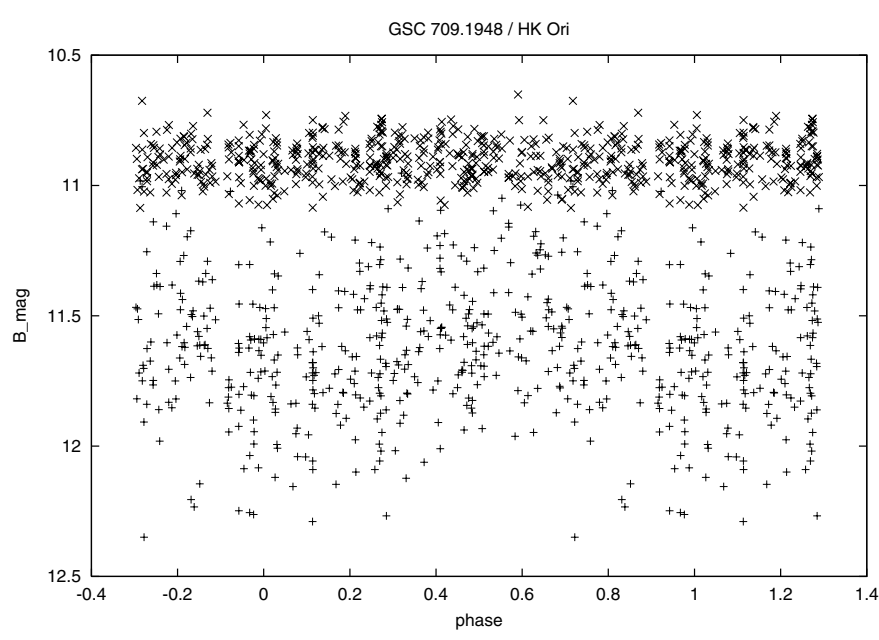

Fig. 15. Light curve of the INSA type variable HK Ori (lower panel) and the constant star GSC 709.1948 vs. phase of the period 2400 days.

\section{Conclusions}

We can summarize the main results of our pilot study as follows:

- The brightness of a single star of $8^{\mathrm{m}}-12^{\mathrm{m}}$ on a scanned blue-sensitive Sonneberg patrol plate can be measured with an accuracy of between 0.07 and $0.12 \mathrm{mag}$, using a twodimensional Gaussian star image fit method and a parabolic calibration fit to HIPPARCOS and TYCHO standard stars.

- This is valid even at plate positions near the edges and corners if the star image is not affected by superposition of nearby neighbours.

- About $7 \%$ of the 216 field stars measured over a time interval of 34 years turned out to be variable with amplitudes of 0.1-0.3 mag, just below the threshold of traditional visual plate inspection methods for variable star research.

- The time scales of most of the new variable stars exceed 1000 days, and are in many cases of the order of 5000-8000 days in the form of slow waves. 


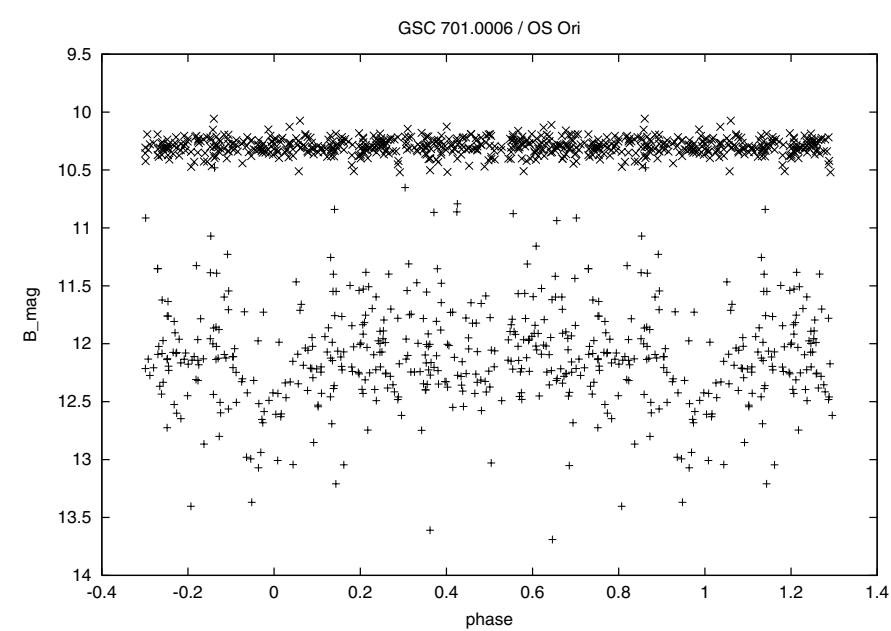

Fig. 16. Light curve of the eclipsing binary OS Ori (lower panel) and the constant star GSC 701.0006 vs. phase of the period 2.383525 .

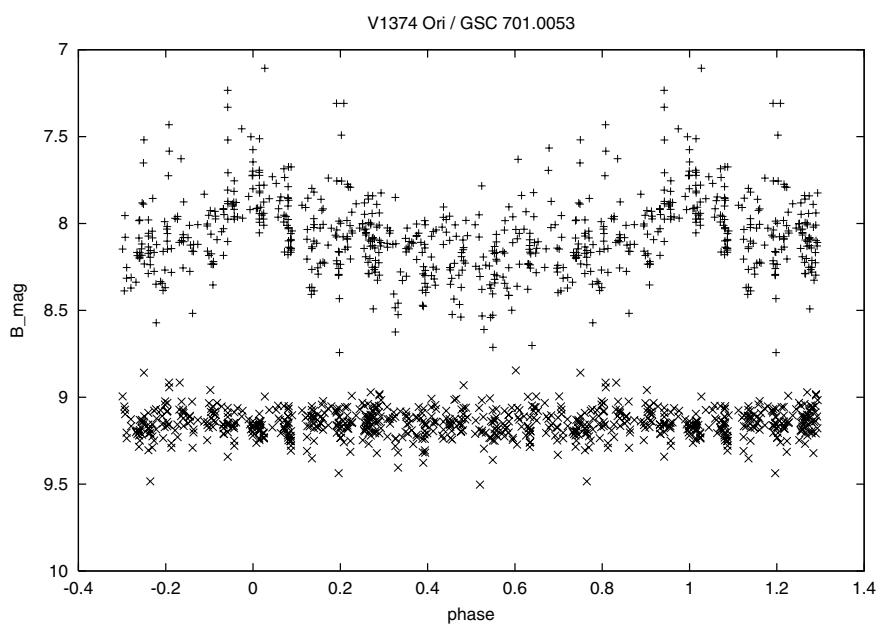

Fig. 17. Light curve of the Be type variable V1374 Ori (upper panel) and the constant star GSC 701.0053 vs. phase of the period 5685 days.

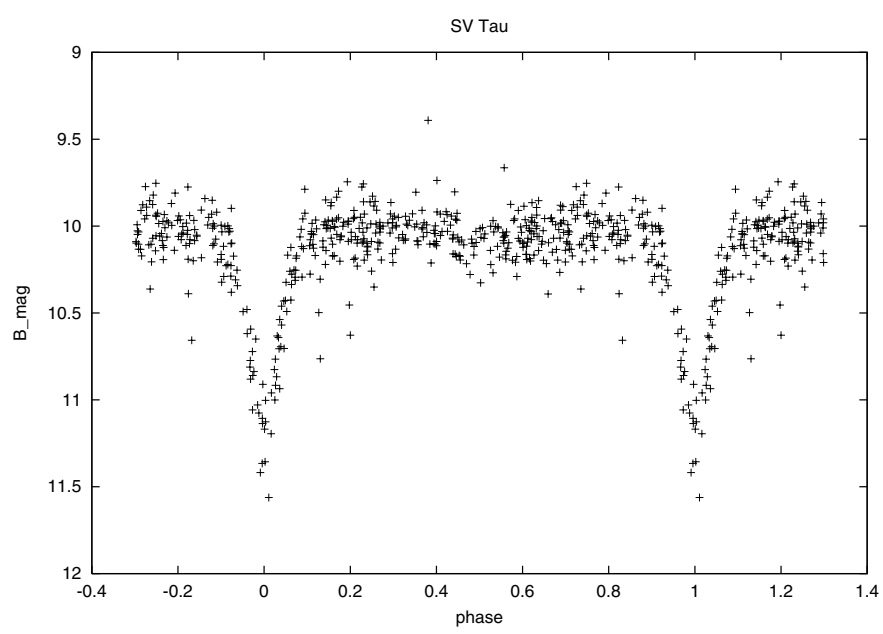

Fig. 18. Light curve of the eclipsing Algol type binary SV Tau vs. phase of the period 2 d 1669051 .

- Nearly $50 \%$ of these new long-term variables exhibit slow drifts indicating periodic or erratic variability at much longer time scales than covered here.

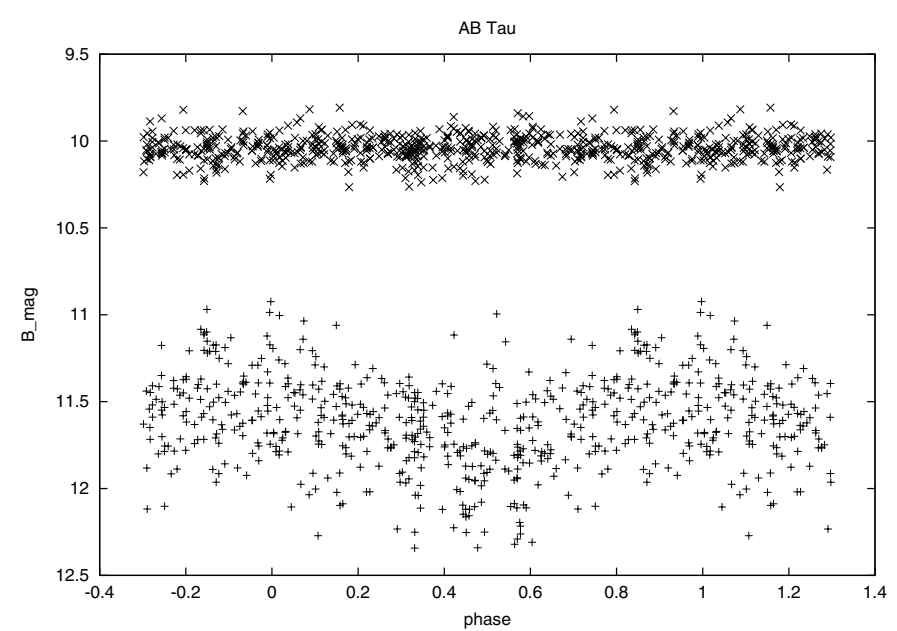

Fig. 19. Light curve of the SRA type variable AB Tau (lower panel) and the constant star GSC 1869.1671 vs. phase of the period 143 days.

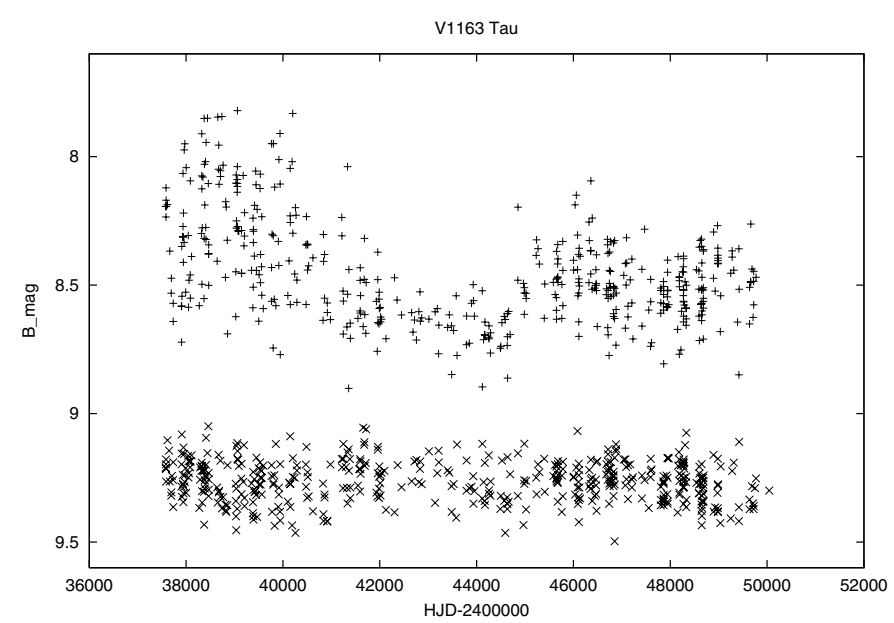

Fig. 20. Light curve of the Be type variable V1163 Tau (upper panel) and the constant star Hip 26845.

- In addition to the field stars we have monitored 23 known variables in the same manner. We achieved improvements in their periods and amplitudes and detected long-term variations (drifts, waves) in about $50 \%$ of them.

- We demonstrate that other presently active projects as MACHO and OGLE which recently have detected a large number of new variable stars do not deliver the same information as our archival patrol plate approach. The methods complement each other.

- We estimate that about 650000 field stars recorded on Sonneberg patrol plates could be studied with our methods. If $7 \%$ of them, as suggested by our pilot study, will show long-term variations with low amplitude, we may, this way, double the number of known variable stars and thus add a population hitherto not investigated.

Acknowledgements. We thank 4pi Systeme - Gesellschaft für Astronomie und Informationstechnologie mbH, Sonneberg, Germany, for financial and logistic support for this project. 


\section{References}

Allen, C. W. 1973, Astrophysical Quantities (London: Third edition, The Athlone Press), 243

Bräuer, H.-J., \& Fuhrmann, B. 1992, Die Sterne, 68, 19

Bräuer, H.-J., Häusele, I., Löchel, K., \& Polko, N. 1999, in TreasureHunting in Astronomical Plate Archives, ed. P. Kroll, C. La Dous, \& H.-J. Bräuer (Frankfurt: H. Deutsch), 70

Heines, A. 1999, in Treasure-Hunting in Astronomical Plate Archives, ed. P. Kroll, C. La Dous, \& H.-J. Bräuer (Frankfurt: H. Deutsch), 205

Jackisch, G. 1963, Veröff. Sternwarte Sonneberg, V, 5, 225

Khopolov, P. N., et al. 1985, General Catalogue of Variable Stars (Moscow: Fourth edition, Nauka Publishing House), Vol. I-IV
Kroll, P. 1999, in Treasure-Hunting in Astronomical Plate Archives, ed. P. Kroll, C. La Dous, \& H.-J. Bräuer (Frankfurt: H. Deutsch), 191

Kroll, P., \& Neugebauer, P. 1993, A\&A, 273, 341

Kukarkin, B. V., et al. 1982, New Catalogue of Suspected Variables (Moscow: Nauka Publishing House)

Richter, G. 1968, Veröff. Sternwarte Sonneberg, VII, 3, 229

Schwarzenberg-Czerny, A. 1989, MNRAS, 241, 153

van Leeuwen, F., Evans, D. W., Grenon, M., et al. 1997, A\&A, 323, L61

Wozniak, P. R., Udalski, A., Szymanski, M., et al. 2002, Acta Astron., 52,129

Zebrun, K., Soszynski, I., Wozniak, P. R., et al. 2001, Acta Astron., 51,317 
N. Vogt et al.: Pilot study on Sonneberg archival plates, Online Material p 1

\section{Online Material}


N. Vogt et al.: Pilot study on Sonneberg archival plates, Online Material p 2

Table 2. Number of measurements, mean B magnitude and scatter $\sigma$ of the constant HIPPARCOS stars.

\begin{tabular}{|c|c|c|c|c|c|c|c|}
\hline $\begin{array}{c}\text { HIPPARCOS } \\
\text { Number }\end{array}$ & $\begin{array}{c}\text { Number of } \\
\text { measurements }\end{array}$ & $\bar{B}$ & $\begin{array}{l}\text { Standard dev. } \bar{\sigma} \\
\quad \text { from } \bar{B}\end{array}$ & $\begin{array}{c}\text { HIPPARCOS } \\
\text { Number }\end{array}$ & $\begin{array}{c}\text { Number of } \\
\text { measurements }\end{array}$ & $\bar{B}$ & $\begin{array}{l}\text { Standard dev. } \bar{\sigma} \\
\text { from } \bar{B}\end{array}$ \\
\hline 23201 & 268 & 8.758 & 0.049 & 26163 & 275 & 10.191 & 0.095 \\
\hline 23209 & 274 & 9.174 & 0.055 & 26196 & 455 & 11.600 & 0.098 \\
\hline 23523 & 389 & 9.310 & 0.057 & 26206 & 414 & 8.724 & 0.092 \\
\hline 23570 & 425 & 10.483 & 0.081 & 26227 & 446 & 11.266 & 0.084 \\
\hline 23634 & 427 & 9.537 & 0.065 & 26239 & 467 & 8.350 & 0.051 \\
\hline 23721 & 431 & 10.694 & 0.105 & 26291 & 414 & 8.379 & 0.105 \\
\hline 23772 & 430 & 9.839 & 0.068 & 26326 & 417 & 8.605 & 0.079 \\
\hline 24035 & 385 & 10.511 & 0.085 & 26335 & 488 & 10.729 & 0.039 \\
\hline 25033 & 420 & 10.049 & 0.094 & 26341 & 474 & 8.920 & 0.090 \\
\hline 25077 & 477 & 8.098 & 0.127 & 26342 & 481 & 10.061 & 0.080 \\
\hline 25123 & 480 & 10.879 & 0.092 & 26356 & 447 & 8.233 & 0.054 \\
\hline 25157 & 433 & 8.006 & 0.076 & 26374 & 456 & 10.875 & 0.088 \\
\hline 25160 & 408 & 7.927 & 0.067 & 26418 & 278 & 8.484 & 0.089 \\
\hline 25185 & 425 & 9.289 & 0.113 & 26419 & 461 & 8.736 & 0.075 \\
\hline 25245 & 466 & 8.152 & 0.066 & 26518 & 462 & 8.269 & 0.064 \\
\hline 25286 & 486 & 9.056 & 0.096 & 26550 & 472 & 9.863 & 0.105 \\
\hline 25294 & 480 & 10.135 & 0.077 & 26555 & 475 & 8.804 & 0.088 \\
\hline 25297 & 484 & 8.614 & 0.090 & 26570 & 452 & 11.213 & 0.101 \\
\hline 25323 & 484 & 8.489 & 0.091 & 26609 & 478 & 9.399 & 0.074 \\
\hline 25326 & 418 & 10.301 & 0.091 & 26618 & 478 & 9.998 & 0.093 \\
\hline 25375 & 484 & 10.365 & 0.096 & 26658 & 399 & 9.115 & 0.089 \\
\hline 25433 & 451 & 7.793 & 0.092 & 26711 & 473 & 8.470 & 0.076 \\
\hline 25445 & 481 & 8.526 & 0.083 & 26729 & 479 & 8.776 & 0.080 \\
\hline 25481 & 485 & 9.152 & 0.118 & 26765 & 438 & 11.076 & 0.085 \\
\hline 25494 & 446 & 10.183 & 0.086 & 26845 & 472 & 9.263 & 0.080 \\
\hline 25512 & 468 & 10.845 & 0.083 & 26852 & 403 & 8.485 & 0.078 \\
\hline 25554 & 449 & 11.055 & 0.091 & 26854 & 466 & 9.738 & 0.092 \\
\hline 25609 & 105 & 9.253 & 0.068 & 26875 & 485 & 9.358 & 0.089 \\
\hline 25614 & 466 & 10.849 & 0.086 & 26946 & 467 & 9.896 & 0.088 \\
\hline 25666 & 485 & 8.879 & 0.117 & 26969 & 467 & 7.977 & 0.065 \\
\hline 25698 & 434 & 10.276 & 0.111 & 26993 & 480 & 9.146 & 0.093 \\
\hline 25711 & 462 & 10.252 & 0.094 & 27312 & 450 & 7.849 & 0.094 \\
\hline 25789 & 454 & 11.365 & 0.117 & 27404 & 464 & 8.896 & 0.049 \\
\hline 25794 & 460 & 7.969 & 0.080 & 27548 & 463 & 9.165 & 0.088 \\
\hline 25801 & 449 & 9.818 & 0.122 & 27605 & 405 & 7.684 & 0.078 \\
\hline 25814 & 402 & 8.817 & 0.069 & 27613 & 466 & 9.702 & 0.107 \\
\hline 25876 & 412 & 10.947 & 0.110 & 27635 & 414 & 10.341 & 0.117 \\
\hline 25904 & 447 & 10.781 & 0.105 & 27676 & 472 & 8.150 & 0.095 \\
\hline 25917 & 420 & 10.227 & 0.120 & 27686 & 475 & 9.620 & 0.095 \\
\hline 25948 & 423 & 8.455 & 0.099 & 27783 & 413 & 8.967 & 0.100 \\
\hline 26028 & 479 & 9.356 & 0.075 & 27798 & 435 & 9.064 & 0.084 \\
\hline 26044 & 479 & 9.994 & 0.086 & 27800 & 472 & 9.231 & 0.095 \\
\hline
\end{tabular}


N. Vogt et al.: Pilot study on Sonneberg archival plates, Online Material p 3

Table 3. Number of measurements, mean B magnitude and scatter $\sigma$ of the constant TYCHO stars.

\begin{tabular}{|c|c|c|c|c|c|c|c|}
\hline $\begin{array}{c}\text { GSC } \\
\text { Number }\end{array}$ & $\begin{array}{c}\text { Number of } \\
\text { measurements }\end{array}$ & $\bar{B}$ & $\begin{array}{l}\text { Standard dev. } \bar{\sigma} \\
\quad \text { from } \bar{B}\end{array}$ & $\begin{array}{c}\text { GSC } \\
\text { Number }\end{array}$ & $\begin{array}{c}\text { Number of } \\
\text { measurements }\end{array}$ & $\bar{B}$ & $\begin{array}{l}\text { Standard dev. } \bar{\sigma} \\
\quad \text { from } \bar{B}\end{array}$ \\
\hline 0700.0205 & 466 & 10.852 & 0.106 & 1873.0388 & 479 & 9.840 & 0.069 \\
\hline 0700.0658 & 271 & 11.757 & 0.090 & 1873.0489 & 399 & 11.717 & 0.085 \\
\hline 0700.0904 & 439 & 11.210 & 0.103 & 1873.0505 & 470 & 10.980 & 0.087 \\
\hline 0700.0931 & 485 & 9.178 & 0.077 & 1873.0519 & 484 & 10.316 & 0.103 \\
\hline 0700.1022 & 473 & 11.055 & 0.110 & 1873.0712 & 452 & 11.625 & 0.104 \\
\hline 0700.1074 & 453 & 11.186 & 0.094 & 1873.0733 & 417 & 11.777 & 0.093 \\
\hline 0700.1124 & 391 & 11.863 & 0.099 & 1873.0742 & 435 & 10.620 & 0.096 \\
\hline 0700.1155 & 462 & 11.360 & 0.124 & 1873.0784 & 461 & 9.809 & 0.085 \\
\hline 0700.1347 & 484 & 9.961 & 0.097 & 1873.0833 & 438 & 11.808 & 0.091 \\
\hline 0700.1538 & 397 & 11.513 & 0.101 & 1874.0147 & 193 & 11.881 & 0.092 \\
\hline 0700.1580 & 465 & 10.995 & 0.104 & 1874.0642 & 468 & 9.744 & 0.091 \\
\hline 0700.1745 & 480 & 10.942 & 0.108 & 1874.1252 & 385 & 11.643 & 0.081 \\
\hline 0701.0006 & 423 & 10.297 & 0.072 & 1874.1261 & 349 & 11.783 & 0.092 \\
\hline 0701.0053 & 482 & 9.151 & 0.082 & 1875.0065 & 461 & 9.278 & 0.104 \\
\hline 0701.0171 & 477 & 9.327 & 0.079 & 2403.0052 & 454 & 11.548 & 0.111 \\
\hline 0701.0356 & 280 & 10.290 & 0.106 & 2403.0181 & 446 & 11.454 & 0.128 \\
\hline 0701.0669 & 477 & 9.838 & 0.082 & 2403.0287 & 440 & 11.319 & 0.097 \\
\hline 0701.0974 & 277 & 9.453 & 0.085 & 2403.0297 & 472 & 10.666 & 0084 \\
\hline 0701.1392 & 472 & 10.828 & 0.107 & 2403.0309 & 457 & 10.661 & 0.100 \\
\hline 0704.0195 & 484 & 9.780 & 0.117 & 2403.0496 & 467 & 10.780 & 0.089 \\
\hline 0704.0605 & 483 & 9.932 & 0.090 & 2403.0654 & 469 & 9.939 & 0.109 \\
\hline 0704.1511 & 465 & 11.197 & 0.121 & 2403.0655 & 458 & 10.177 & 0.093 \\
\hline 0705.0092 & 313 & 12.160 & 0.066 & 2403.0657 & 456 & 11.212 & 0.109 \\
\hline 0705.0366 & 391 & 11.714 & 0.079 & 2403.0736 & 426 & 11.676 & 0.113 \\
\hline 0705.0442 & 102 & 10.397 & 0.081 & 2403.0758 & 325 & 11.271 & 0.105 \\
\hline 0705.0920 & 479 & 9.853 & 0.100 & 2403.0857 & 443 & 11.248 & 0.103 \\
\hline 0708.0577 & 485 & 9.727 & 0.090 & 2403.0963 & 414 & 11.630 & 0.120 \\
\hline 0708.1646 & 479 & 9.867 & 0.087 & 2403.1062 & 452 & 11.656 & 0.124 \\
\hline 0708.1710 & 453 & 9.206 & 0.094 & 2403.1246 & 384 & 12.100 & 0.111 \\
\hline 0709.0030 & 422 & 9.664 & 0.102 & 2403.1379 & 407 & 11.391 & 0.117 \\
\hline 0709.1150 & 400 & 11.607 & 0.069 & 2404.0110 & 464 & 11.067 & 0.091 \\
\hline 0709.1571 & 375 & 11.555 & 0.071 & 2404.0128 & 427 & 9.851 & 0.107 \\
\hline 0709.1948 & 431 & 10.904 & 0.078 & 2404.0204 & 369 & 11.680 & 0.086 \\
\hline 0709.2061 & 455 & 11.232 & 0.093 & 2404.0231 & 192 & 11.640 & 0.098 \\
\hline 0714.0247 & 477 & 9.327 & 0.079 & 2404.0358 & 449 & 11.131 & 0.093 \\
\hline 1840.0262 & 317 & 11.725 & 0.038 & 2404.0403 & 420 & 11.625 & 0.112 \\
\hline 1853.0315 & 429 & 10.949 & 0.111 & 2404.0564 & 454 & 11.098 & 0.094 \\
\hline 1853.0713 & 428 & 11.007 & 0.095 & 2404.0578 & 467 & 10.518 & 0.095 \\
\hline 1857.1561 & 431 & 10.954 & 0.095 & 2404.0746 & 214 & 11.832 & 0.075 \\
\hline 1857.1645 & 431 & 10.112 & 0.081 & 2404.0821 & 446 & 10.809 & 0.096 \\
\hline 1859.0283 & 252 & 12.122 & 0.067 & 2404.0993 & 439 & 11.591 & 0.101 \\
\hline 1859.1338 & 403 & 9.775 & 0.094 & 2404.1175 & 455 & 11.268 & 0.105 \\
\hline 1860.0021 & 423 & 10.666 & 0.099 & 2405.0063 & 420 & 9.421 & 0.074 \\
\hline 1860.0211 & 130 & 11.414 & 0.078 & 2405.0620 & 429 & 11.579 & 0.071 \\
\hline 1860.0229 & 240 & 11.105 & 0.091 & 2405.0856 & 458 & 9.982 & 0.110 \\
\hline 1860.0362 & 443 & 10.249 & 0.090 & 2405.1338 & 384 & 10.861 & 0.075 \\
\hline 1860.0486 & 198 & 11.549 & 0.081 & 2405.1617 & 409 & 11.478 & 0.100 \\
\hline 1860.0726 & 310 & 11.106 & 0.099 & 2405.1747 & 403 & 11.217 & 0.086 \\
\hline 1860.0785 & 300 & 11.421 & 0.081 & 2406.0439 & 456 & 10.937 & 0.103 \\
\hline 1860.1233 & 436 & 9.663 & 0.093 & 2406.0529 & 352 & 11.812 & 0.053 \\
\hline 1860.1260 & 427 & 9.452 & 0.079 & 2406.0677 & 458 & 11.155 & 0.108 \\
\hline 1860.1270 & 432 & 10.668 & 0.093 & 2407.0056 & 378 & 8.963 & 0.099 \\
\hline 1869.1223 & 468 & 11.341 & 0.087 & 2407.1282 & 412 & 11.130 & 0.116 \\
\hline 1869.1325 & 481 & 8.619 & 0.093 & 2408.0323 & 364 & 11.651 & 0.127 \\
\hline 1869.1671 & 475 & 10.041 & 0.074 & 2408.0619 & 336 & 11.617 & 0.085 \\
\hline 1869.1689 & 474 & 11.278 & 0.098 & 2409.0281 & 409 & 10.692 & 0.116 \\
\hline 1870.1592 & 129 & 12.089 & 0.095 & 2409.0423 & 392 & 9.991 & 0.094 \\
\hline 1873.0139 & 468 & 11.299 & 0.098 & & & & \\
\hline
\end{tabular}

